\title{
Article \\ Detoxification and Excretion of Trichothecenes in Transgenic Arabidopsis thaliana Expressing Fusarium graminearum Trichothecene 3-O-acetyltransferase
}

\author{
Guixia Hao *, Susan McCormick (D), Helene Tiley and Thomas Usgaard
}

Citation: Hao, G.; McCormick, S.; Tiley, H.; Usgaard, T. Detoxification and Excretion of Trichothecenes in Transgenic Arabidopsis thaliana Expressing Fusarium graminearum Trichothecene 3-O-acetyltransferase. Toxins 2021, 13, 320. https://doi.org/ $10.3390 /$ toxins 13050320

Received: 22 April 2021

Accepted: 27 April 2021

Published: 29 April 2021

Publisher's Note: MDPI stays neutral with regard to jurisdictional claims in published maps and institutional affiliations.

Copyright: (c) 2021 by the authors. Licensee MDPI, Basel, Switzerland. This article is an open access article distributed under the terms and conditions of the Creative Commons Attribution (CC BY) license (https:// creativecommons.org/licenses/by/ $4.0 /)$.
USDA, Agricultural Research Service, Mycotoxin Prevention and Applied Microbiology Research Unit, National Center for Agricultural Utilization Research, 1815 N. University street, Peoria, IL 61604, USA; susan.mccormick@usda.gov (S.M.); helene.tiley@usda.gov (H.T.); thomas.usgaard@usda.gov (T.U.)

* Correspondence: guixia.hao@usda.gov; Tel.: +1-309-681-6520

\begin{abstract}
Fusarium graminearum, the causal agent of Fusarium head blight (FHB), produces trichothecenes including deoxynivalenol (DON), nivalenol (NIV), and 3,7,15-trihydroxy-12,13-epoxytrichothec-9-ene (NX-3). These toxins contaminate grains and cause profound health problems in humans and animals. To explore exploiting a fungal self-protection mechanism in plants, we examined the ability of $F$. graminearum trichothecene 3-O-acetyltransferase (FgTri101) to detoxify several key trichothecenes produced by F. graminearum: DON, 15-ADON, NX-3, and NIV. FgTri101 was cloned from F. graminearum and expressed in Arabidopsis plants. We compared the phytotoxic effects of purified DON, NIV, and NX-3 on the root growth of transgenic Arabidopsis expressing FgTri101. Compared to wild type and GUS controls, FgTri101 transgenic Arabidopsis plants displayed significantly longer root length on media containing DON and NX-3. Furthermore, we confirmed that the FgTri101 transgenic plants acetylated DON to 3-ADON, 15-ADON to 3,15-diADON, and NX-3 to NX-2, but did not acetylate NIV. Approximately $90 \%$ of the converted toxins were excreted into the media. Our study indicates that transgenic Arabidopsis expressing FgTri101 can provide plant protection by detoxifying trichothecenes and excreting the acetylated toxins out of plant cells. Characterization of plant transporters involved in trichothecene efflux will provide novel targets to reduce FHB and mycotoxin contamination in economically important plant crops.
\end{abstract}

Keywords: Fusarium graminearum; Arabidopsis; transgenic plants; trichothecene; deoxynivalenol; nivalenol; NX-3; detoxification; excretion

Key Contribution: Transgenic Arabidopsis expressing FgTri101 not only converted DON to 3-ADON, but also detoxified 15-ADON and NX-3. More importantly, the acetylated trichothecenes were excreted to the media to protect the plants. This study suggests that FgTri101 transgenic plants employ trichothecene detoxification and efflux systems for self-protection.

\section{Introduction}

Mycotoxins are secondary metabolites produced by fungi and pose serious threats to human and animal health by contaminating food and feed supplies. Fusarium species produce a variety of trichothecenes, including T-2 toxin, 4,15-diacetoxyscirpenol (DAS), nivalenol (NIV), deoxynivalenol (DON), and its acylated derivatives 3-acetyldeoxynivalenol (3-ADON) and 15-acetyldeoxynivalenol (15-ADON). Trichothecenes produced by the fungal pathogen F. graminearum, a major causal agent for Fusarium head blight (FHB), contaminate wheat, barley, and other small grains. Trichothecenes have multiple inhibitory effects on eukaryotic cells, including DNA and RNA synthesis, protein translation and elongation, mitochondria translation, and cell division [1,2]. Consumption of trichothecenecontaminated products causes immunotoxicity and cytotoxicity in humans and animals. In the United States, the Food and Drug Administration has issued advisory levels for 
DON in cereals and associated products for human consumption (1 ppm) and animal feed (5-10 ppm).

Trichothecenes play an important role during F. graminearum and host interactions. DON is a critical factor for FHB spread in wheat. Mutants deficient in DON production are restricted to inoculated florets and cannot pass the rachis node to spread to adjacent spikes $[3,4]$. On the other hand, transgenic plants expressing genes that detoxify DON increase DON and FHB resistance. For example, transgenic Arabidopsis and wheat plants expressing a barley UDP-glucosyltransferase, which converts DON to the less toxic DON3-O-glucoside (D3G), exhibit resistance to DON [5,6]. Transgenic wheat plants expressing Fhb7, encoding a glutathione S-transferase, both detoxify trichothecenes and enhance FHB resistance [7]. Thus, the utilization of trichothecene detoxification is a promising approach to reduce FHB and mycotoxin contamination.

The gene cluster and biosynthetic pathways of trichothecenes are well characterized in Fusarium species. A core TRI gene cluster contains 12 TRI genes located on chromosome 2. TRI5, the first gene for trichothecene biosynthesis, and TRI6, the regulatory gene for trichothecene biosynthesis, reside in the TRI gene cluster [8]. For self-protection, Fusarium species employ a transporter (TRI12) to pump toxins out of the fungal cells, and a trichothecene 3-O-acetyltransferase (TRI101) to reduce toxicity of isotrichodermol and other intermediates during the biosynthesis of trichothecenes [9]. Although TRI101 is located outside of the TRI gene cluster, the expression of TRI101 is regulated by TRI6. In addition, Tri101 catalyzes the conversion of toxic Fusarium trichothecenes, such as DON, T-2 toxin, and HT-2 toxin, into less toxic C3-acetylated products [9].

The detoxification ability of TRI101 has been exploited to reduce trichothecenes and FHB, and studies have shown that transgenic plants expressing TRI101 convert DON to 3-ADON and have increased tolerance to trichothecenes such as DAS or DON [10-13]. However, most of these studies expressed F. sporotrichioides TRI101 (FsTRI101), and transgenic plants expressing FsTRI101 did not significantly increase wheat and barley resistance toward F. graminearum [11,12]. Further studies demonstrated that the proteins encoded by FgTri101 and its ortholog FsTri101 share about 80\% identity. FgTri101 is much more effective in converting DON to 3-ADON in comparison to FsTri101 [14]. Partly because of this reason, transgenic wheat plants expressing FsTRI101 only displayed partial resistance to FHB caused by F. graminearum [11]. Therefore, it is necessary to reexamine the potential to reduce FHB and trichothecenes contamination in transgenic plants expressing FgTri101. In addition, three genetically distinct populations of $F$. graminearum have been characterized as primary causal agents of FHB in North America [15]: NA1, which predominantly produces $15-\mathrm{ADON}$; NA2, a more aggressive population that produces $3-\mathrm{ADON}$; and a recently identified population, referred to as NA3, which produces the trichothecene ana$\log , 3 \alpha$-acetoxy, 7,15-dihydroxy-12,13-epoxytrichothec-9-ene (NX-2). Although the three populations produce different trichothecene phenotypes (chemotypes) in liquid culture, in planta the trichothecenes are primarily found in their deacetylated form. Both 3-ADON and 15-ADON are converted to DON, and NX-2 to 3,7,15-trihydroxy-12,13-epoxytrichothec9-ene (NX-3). The toxic effects of the NX-3 toxin in inducing cytotoxicity and intestinal inflammatory are comparable to DON [16]. Control of FHB and mycotoxin contamination remains a challenge due to a lack of completely resistant varieties, a complicated fungal infection process, and emerging fungicide-resistant strains. In order to identify a successful strategy to reduce FHB and mycotoxin contamination, it is important to determine whether FgTri101 can effectively detoxify the novel trichothecene NX-3 as well as DON.

Since wheat transformation and regeneration are time-consuming processes, in this study, we generated transgenic Arabidopsis plants expressing FgTri101. The goal of this study was to determine whether the transgenic Arabidopsis plants expressing the FgTri101 gene can increase resistance to several key trichothecenes. We also examined whether FgTri101 transgenic plants can convert DON, 15-ADON, NX-3, and NIV to their acetylated forms. 


\section{Results}

\subsection{Generation of Transgenic Arabidopsis Expressing FgTri101}

Arabidopsis Col-0 was transformed with the plasmid pBinARS/plus-FgTri101, driven by a double CaMV35S promoter (Figure S1). A total of $20 \mathrm{~T}_{0}$ plants were obtained on kanamycin-containing selection media. The presence of the FgTri101 gene was confirmed in nine independent $\mathrm{T}_{0}$ transgenic Arabidopsis plants by PCR amplification (Figure S1). The expression of FgTri101 in these transgenic plants was determined by RT-qPCR. The transgenic plant Tri101-12 displayed the highest expression level of FgTri101 (765-fold), followed by Tri101-17 (21-fold) and Tri101-8 (14-fold), relative to the lowest expression plant Tri101-10 that had $C_{t}$ values of $22.8 \pm 0.3$ (Figure 1). Four transgenic lines, Tri101-8, 10,12 , and 17 , were selected for further study.

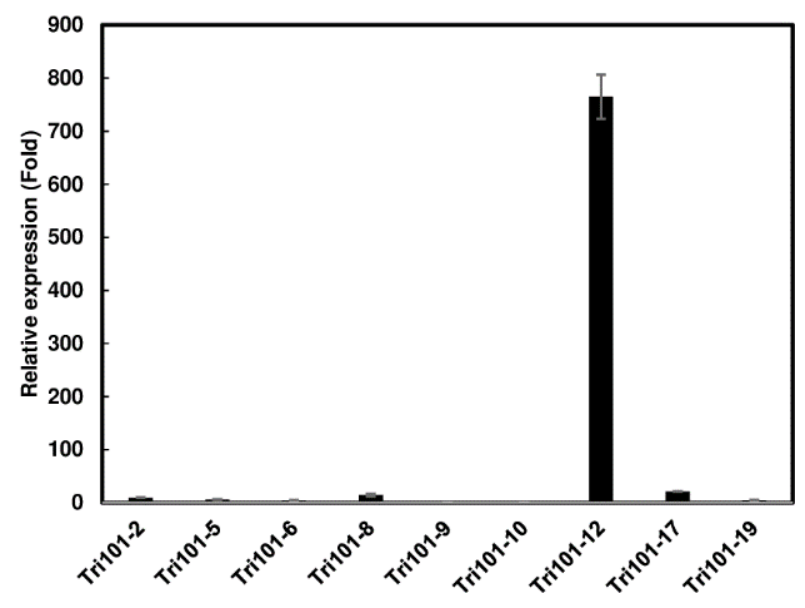

Figure 1. Expression of FgTri101 in independent transgenic Arabidopsis plants. The expression of FgTri101 was normalized to the expression of Arabidopsis elongation factor 1-alpha (EF1 $\alpha$ ). The relative gene expression was calculated from the $2^{-\Delta \Delta C t}$ values of a sample versus Arabidopsis FgTri101-10, which had the lowest expression among the tested samples. The samples were run in three biological replicates with three technical replicates. Bars represent the average means from three biological replicates and their standard deviations.

The $T_{2}$ seeds collected from the $T_{1}$ transgenic plants (Tri101-8, 10,12, and 17) were examined for segregation on a kanamycin-containing selection medium. These transgenic lines displayed no segregation for kanamycin resistance and therefore were considered homozygous. Furthermore, we determined the copy number of FgTri101 in three transgenic homozygous lines (TRI101-8- $\mathrm{T}_{3}, 12-\mathrm{T}_{3}$, and $17-\mathrm{T}_{3}$ ) and nine hemizygous $\mathrm{T}_{0}$ TRI101 plants by quantitative real-time PCR. The Arabidopsis gene 4-hydroxyphenypyruvate dioxygenase $(4 H P P D)$ was used as an endogenous reference. The amplification efficiency of the target gene FgTri101 and the Arabidopsis 4HPPD was $91 \%$ and $101.9 \%$ respectively. Using the ratio formula calculation, one transgenic plant, TRI101-6, appeared to have two insertions using DNA from hemizygous $\mathrm{T}_{0}$ plants (Table $\mathrm{S} 1$ ). We estimated a single FgTri101 insertion was present in the rest of the Arabidopsis transgenic lines (Table S1).

\subsection{Enhanced Root Growth of Transgenic Arabidopsis Plants Expressing FgTri101 on Media Containing Trichothecenes}

Since in planta trichothecenes are primarily found in their deacetylated forms, the germination and growth of transgenic Arabidopsis seeds were assessed on MS media containing DON, NX-3, and NIV, respectively, to evaluate the protective effect provided by FgTri101. On the media containing $10 \mathrm{mg} / \mathrm{L}$ DON or NX-3, the control Arabidopsis Col-0 and GUS transgenic seeds germinated, but displayed small yellow cotyledons, necrotic lesions on leaves, and very short roots. In contrast, the plants expressing FgTri101 exhibited green leaves and long shoots and roots (Figures 2 and S2). Statistical analyses showed that all four FgTri101 transgenic lines displayed a significantly longer root length 
compared to the Arabidopsis Col-0 and GUS controls $(p<0.001)$ on DON and NX-3 plates (Figures 3 and S3). However, on the media containing $10 \mathrm{mg} / \mathrm{L}$ NIV, FgTri101, Arabidopsis Col-0, and GUS transgenic lines displayed similar phenotypes: green leaves and long roots (Figure 2 and Figure S2). The roots of Arabidopsis Col-0 and GUS transgenic plants on media containing NIV were significantly longer compared with those on the media containing DON or NX-3 (Figure S3). These results suggest that expression of FgTri101 did not provide plant protection toward NIV, and that NIV is less phytotoxic. Taken together, our observations indicate that the expression of FgTri101 in the transgenic Arabidopsis plants showed enhanced resistance to DON and NX-3, but not to NIV.

(a) DON

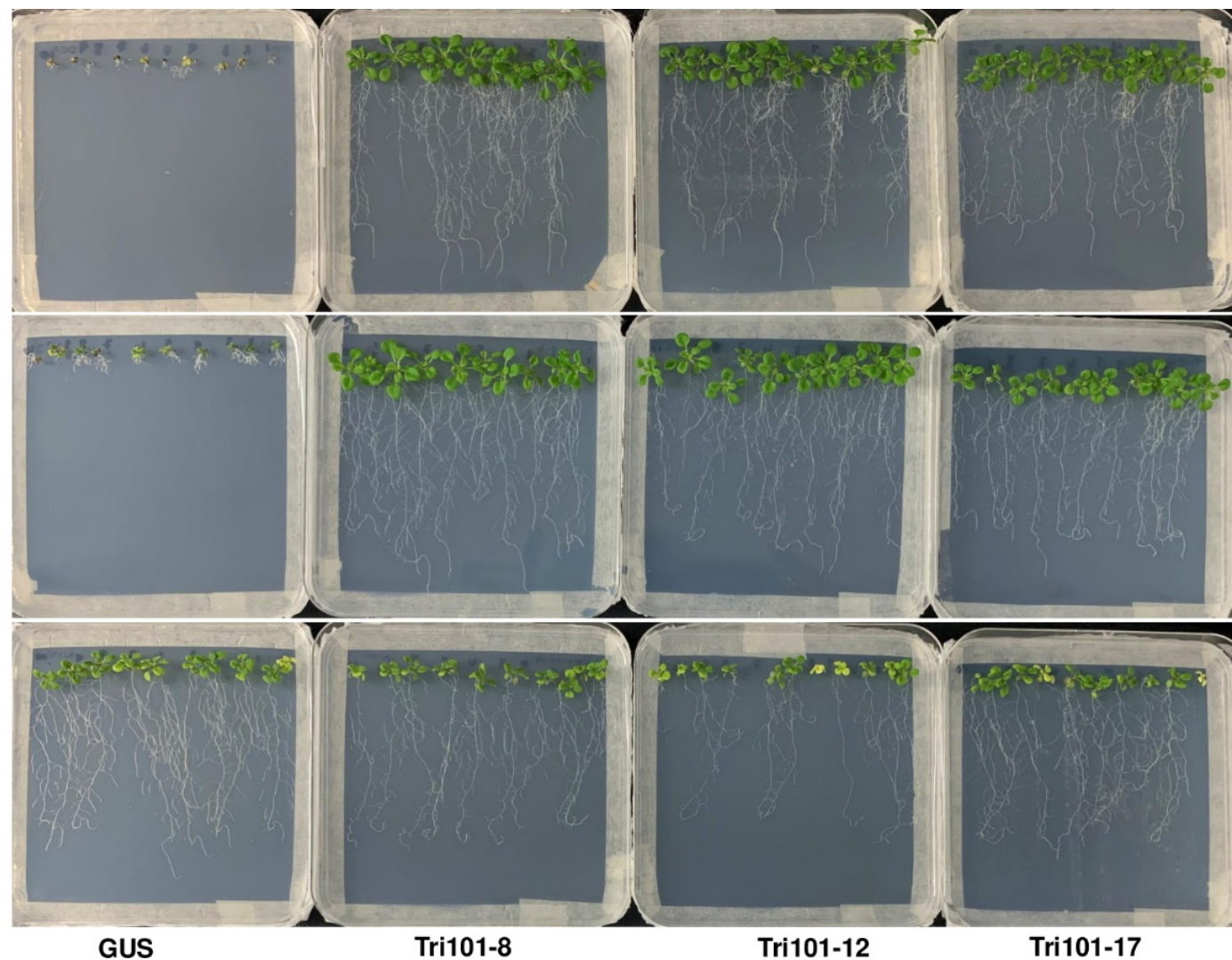

Figure 2. Arabidopsis seedling growth on MS media containing different trichothecenes (10 mg/L). (a) DON; (b) NX-3; and (c) NIV. Three transgenic lines (FgTri101-8, 12, and 17) and a control line expressing GUS were used. The photographs were taken after a two-week incubation.

(a)

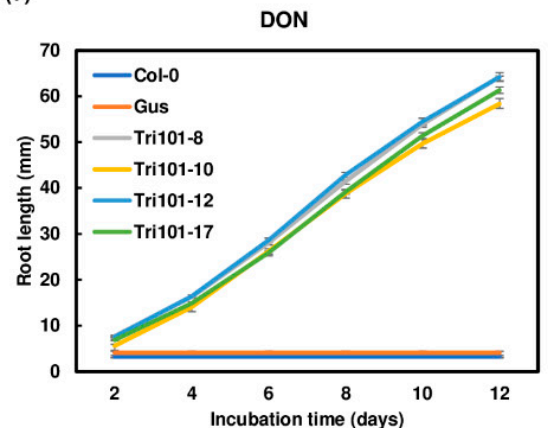

(b)

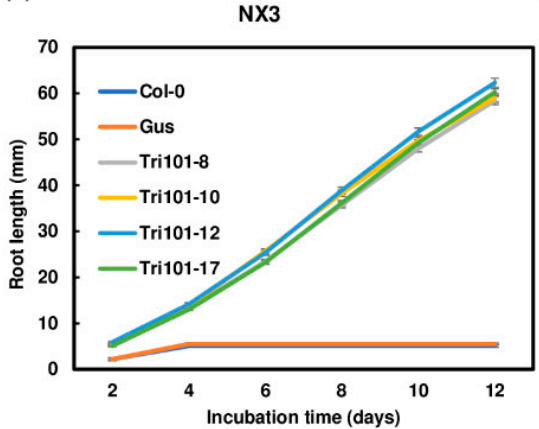

(c)

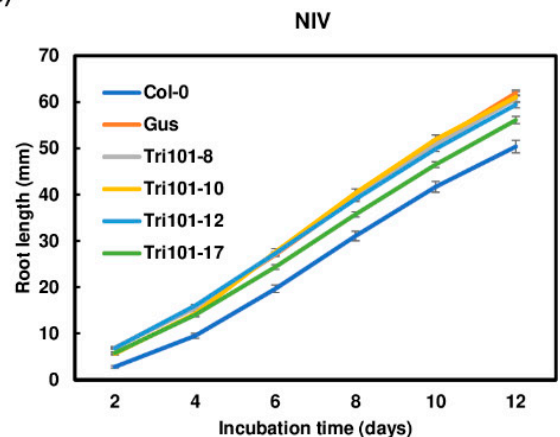

Figure 3. Root length comparison of the Arabidopsis plants expressing FgTri101 grown on trichothecene-containing media. Seeds were germinated on half-strength MS media containing $10 \mathrm{mg} / \mathrm{L}$ of the individual toxins. (a) DON; (b) NX-3; and (c) NIV. Root lengths were measured every two days for two weeks. Each treatment contained 36-40 seedlings. 


\subsection{Acetylation and Excretion of Trichothecenes by Transgenic Arabidopsis Seedlings Expressing FgTri101}

To confirm the acetylation function of FgTri101 in transgenic Arabidopsis, we treated Arabidopsis seedlings with $50 \mathrm{mg} / \mathrm{L}$ DON, 15-ADON, NX-3, or NIV in liquid half-strength MS media. After seedlings were treated for 2 days with individual toxins, the concentrations of trichothecenes were measured in Arabidopsis seedlings and in the media. Our results showed that DON was efficiently converted to 3-ADON in the transgenic seedlings expressing FgTri101, whereas no 3-ADON was detected in wild-type Col-0 and GUS transgenic seedlings (Figure 4a). This observation confirmed that the expression of FgTri101 in transgenic Arabidopsis can convert DON to 3-ADON. Interestingly, we detected 3-ADON in the media growing FgTri101 transgenic seedlings when DON was added to the media (Figure $4 \mathrm{~b}$ ). Surprisingly, over $96 \%$ of $3-\mathrm{ADON}$ was detected in the media out of the total converted 3-ADON (Figure 4c). These results suggest that Arabidopsis transporter/transporters can efficiently pump out 3-ADON following the FgTri101 detoxification of DON.

(a)

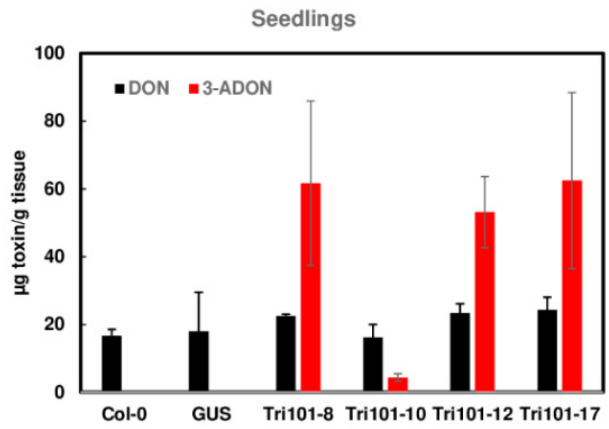

(c)

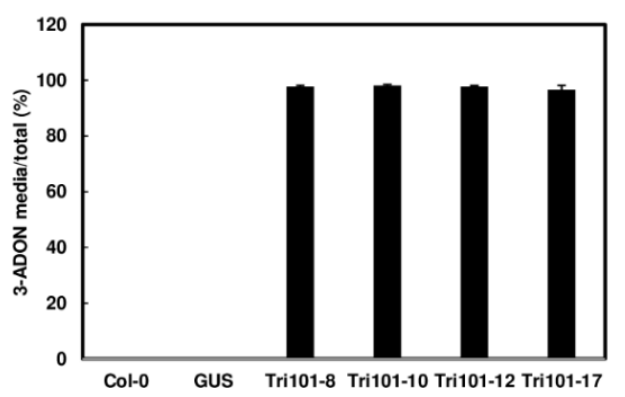

(b)

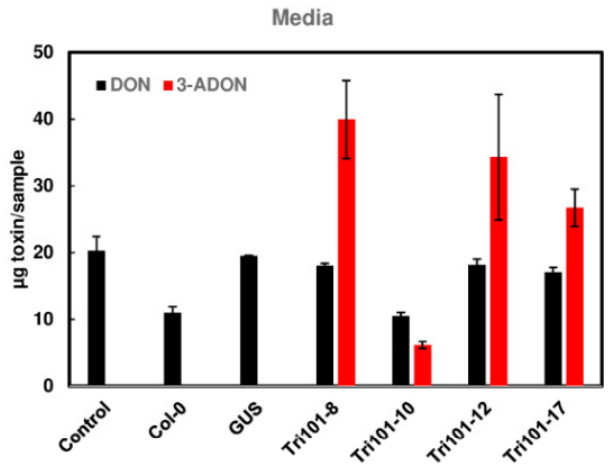

Figure 4. Conversion of DON to 3-ADON and excretion of 3-ADON by FgTri101 transgenic Arabidopsis. (a) DON and 3-ADON in Arabidopsis seedlings after treatment with $50 \mathrm{mg} / \mathrm{L} \mathrm{DON}$ for 2 days. All plant tissues were extracted for toxins. (b) DON and 3-ADON in the media after Arabidopsis seedlings were treated with $50 \mathrm{mg} / \mathrm{L}$ DON for 2 days. Media without plant seedlings served as a control. Toxins in $1.4 \mathrm{~mL}$ aliquots from $20 \mathrm{~mL}$ media were measured and presented. (c) 3-ADON in the media in comparison to total 3-ADON in the plant and media. Percentage of excreted 3-ADON: 3-ADON media / (3-ADON media + plant) $\times 100$.

When 15-ADON was added, we observed reduced 15-ADON in FgTri101 transgenic Arabidopsis compared to GUS transgenic Arabidopsis, but we did not detect 3,15-diADON in the FgTri101 seedlings. In a repeated experiment, 3,15-diADON was detected in only a few seedling samples from each transgenic line, suggesting the conversion of 15-ADON to 3,15-diADON (Figures 5a and S4). In contrast, a large amount of 3,15-diADON (98\%) was detected in the media when 15-ADON was added to FgTri101 transgenic seedling cultures (Figure 5b). 3,15-diADON was not detected in wild-type Col-0 and GUS transgenic plants or in their growth media. These results indicate that the transgenic Arabidopsis seedlings expressing FgTri101 convert 15-ADON to 3,15-ADON and pump most of it to the media. 
(a)

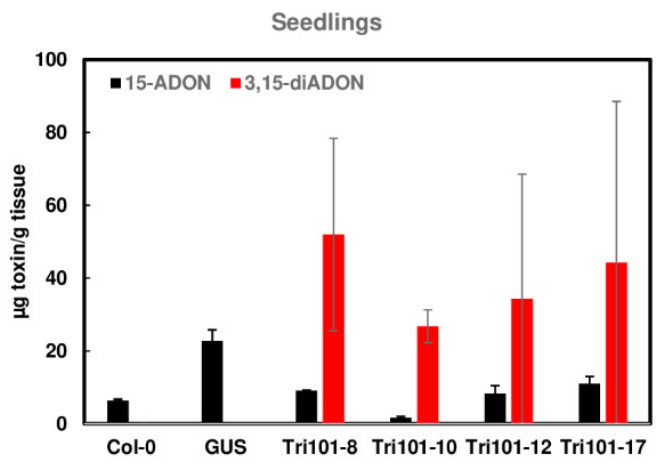

(c)

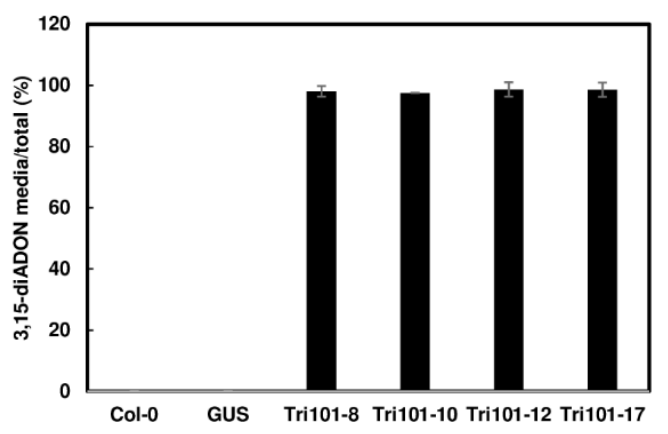

(b)

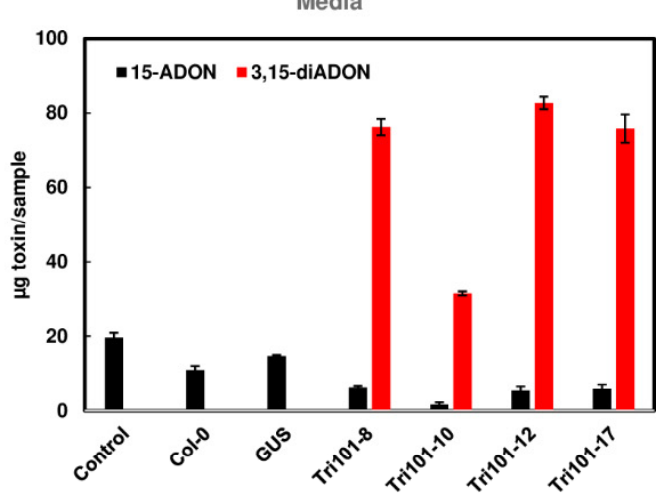

Figure 5. Conversion of 15-ADON to 3,15-diADON and excretion of 3,15-diADON by FgTri101 transgenic Arabidopsis. (a) 15-ADON, and 3,15-diADON in Arabidopsis seedlings after treatment with $50 \mathrm{mg} / \mathrm{L}$ 15-ADON for 2 days. (b) 15-ADON and 3,15-diADON in the media after Arabidopsis seedlings were treated with $50 \mathrm{mg} / \mathrm{L} \mathrm{15-ADON}$ for 2 days. Media without plant seedlings served as a control. Toxins in the media were measured in $1.4 \mathrm{~mL}$ aliquots from $20 \mathrm{~mL}$ of media and presented. (c) 3,15-diADON in the media in comparison to 3,15-diADON in the plant and media. Percentage of excreted 3,15-diADON: 3,15-diADON media/ (3,15-diADON media + plant) $\times 100$.

Similarly, NX-3 was converted to NX-2 in transgenic Arabidopsis seedling expressing FgTri101, but there was no conversion in wild-type Col-0 and GUS seedlings (Figure 6a). NX-2 was excreted to the media (Figure $6 \mathrm{~b}$ ), and about $90 \%$ of NX-2 was exported to the media (Figure 6c).

However, 3-ANIV was not detected in FgTri101 transgenic seedlings. The added NIV was observed in Arabidopsis seedlings expressing GUS or Tri101 (Figure S5), suggesting that Arabidopsis seedlings can uptake NIV, but have no ability to convert it to 3-ANIV. This explained the growth results that transgenic Arabidopsis expressing FgTri101, wild-type Col-0, or GUS had similar phenotypes on NIV-supplemented media.

Taken together, our results suggest that Arabidopsis expressing FgTri101 can detoxify DON, 15-ADON, and NX-3, and efficiently pump out acetylated trichothecenes to protect the transgenic plants. However, Arabidopsis expressing FgTri101 provides no protection against NIV.

\subsection{Transgenic Arabidopsis Expressing FgTri101 Accumulated Higher Weight with Addition of Trichothecenes}

To examine the protective effect of transgenic Arabidopsis expressing FgTri101, the total weights of Arabidopsis seedlings were determined after being treated with $50 \mathrm{mg} / \mathrm{L}$ DON, 15-ADON, NX-3, or NIV in liquid MS media. After treatments of $48 \mathrm{~h}$ with DON, 15-ADON, or NX-3, the total weights of the FgTri101 transgenic lines were significantly higher compared to the GUS transgenic controls (Figure 7a-c). However, no significant difference was observed between the NIV-treated GUS and FgTri101 seedlings (Figure 7d). These results suggest that FgTri101 can provide plant protection from trichothecenes DON and NX-3, but not from NIV produced by F. graminearum. 
(a)

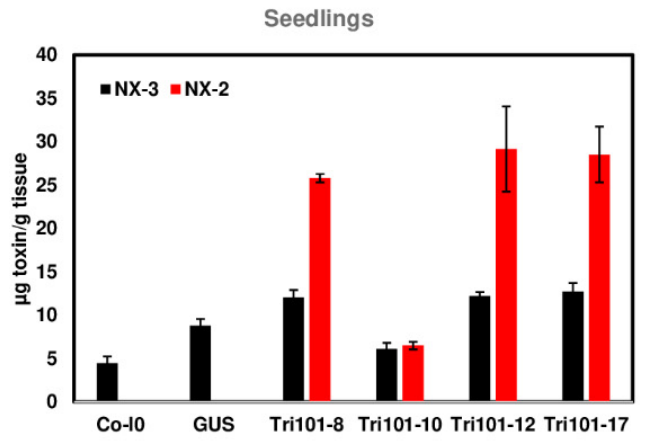

(c)

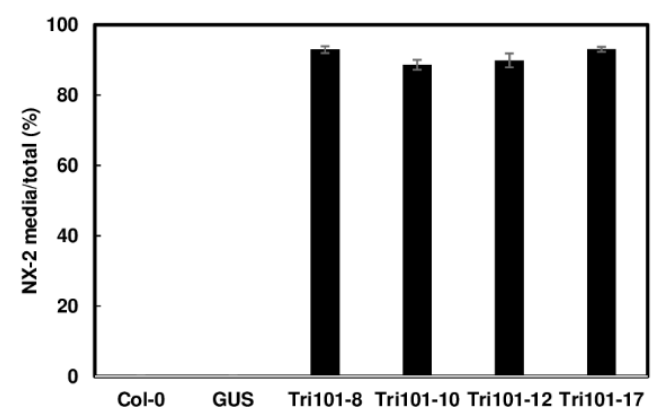

(b)

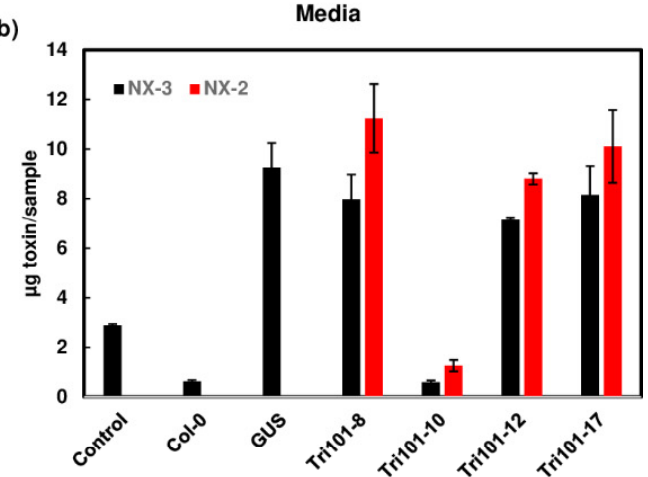

Figure 6. Conversion of NX-3 to NX-2 and excretion of NX-2 by FgTri101 transgenic Arabidopsis. (a) NX-3 and NX-2 in the Arabidopsis seedlings after treatment with 50 mg/L NX-3 for 2 days. (b) NX-3 and NX-2 in the media after Arabidopsis seedlings were treated with $50 \mathrm{mg} / \mathrm{L} \mathrm{NX-3}$ for 2 days. Media without plant seedlings served as a control. Toxins in the media were measured in $1.4 \mathrm{~mL}$ aliquots from $20 \mathrm{~mL}$ of media and presented. (c) NX-2 in media in comparison to NX-3 in plant and media. Percentage of excreted NX-2: NX-2 media/ (NX-2 media + plant) $\times 100$.

(a)

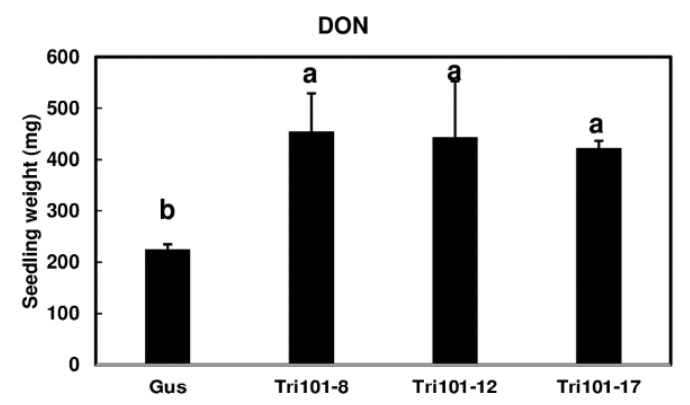

(c)

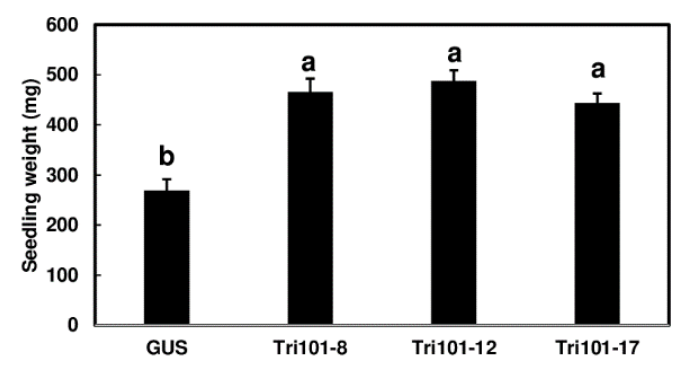

(b)

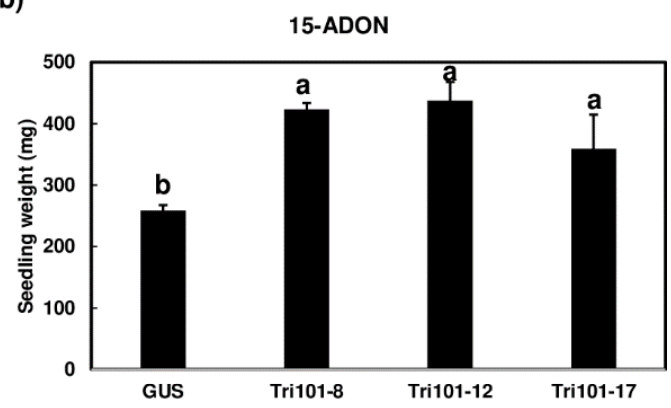

(d)

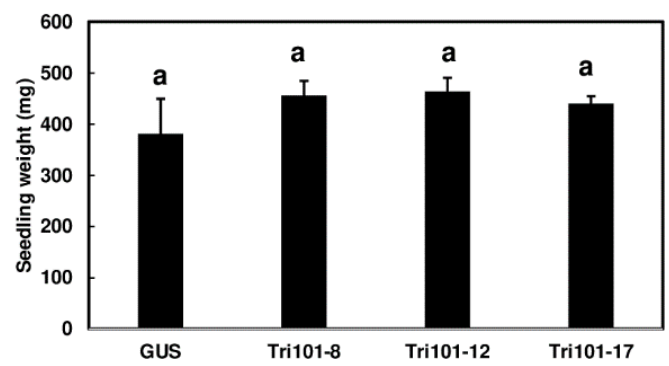

Figure 7. Weight comparison in transgenic Arabidopsis seedlings expressing FgTri101 and GUS with trichothecenes treatments. Arabidopsis seedlings were treated with individual trichothecenes (50 mg/L) for 2 days: (a) DON; (b) 15-ADON; (c) NX-3; and (d) NIV. The data were analyzed by one-way ANOVA and Tukey's post hoc test using JMP. Bars with different letters indicate statistically significant differences. 


\subsection{Morphology of Transgenic Arabidopsis Plants Expressing FgTri101}

To assess whether the transgenic expression of FgTri101 affects Arabidopsis growth and morphology, FgTri101 transgenic lines (Tri-8, 12, and 17) and the GUS transgenic control were germinated on MS media containing kanamycin and transferred to soil. The flowering time, rosette leaf number, plant height, and number of shoots were evaluated. All three FgTri101 expression lines did not show a significantly different flowering time, plant height, and number of shoots compared with the GUS control (Table 1). However, the FgTri101 line 12, which had the highest expression level of FgTri101, displayed significantly fewer rosette leaf numbers compared with the GUS control. Nevertheless, the expression of the FgTri101 gene without extremely high levels did not significantly affect the overall Arabidopsis morphology.

Table 1. Morphological comparison of transgenic Arabidopsis plants expressing FgTri101 and GUS.

\begin{tabular}{|c|c|c|c|c|}
\hline Genotypes & Flower Time (days) & Rosette Leaf Number ${ }^{a}$ & Plant Height $(\mathrm{cm})^{b}$ & Shoot Number ${ }^{b}$ \\
\hline GUS & 27 & $5.4 \pm 0.52$ & $32.6 \pm 2.29$ & $5.6 \pm 1.30$ \\
\hline Tri101-8 & 27 & $5.1 \pm 0.35$ & $29.5 \pm 2.09$ & $6.5 \pm 1.41$ \\
\hline Tri101-12 & 27 & $4.6^{*} \pm 0.50$ & $31.5 \pm 3.85$ & $6.5 \pm 1.69$ \\
\hline Tri101-17 & 27 & $5.0 \pm 0.53$ & $31.5 \pm 2.19$ & $5.6 \pm 1.19$ \\
\hline
\end{tabular}

${ }^{a}$ The number of the rosette leaves was counted at 17 days after plants were transferred to soil. ${ }^{b}$ The plant height and shoot were measured when plants matured at about 40 days grown in soil. ${ }^{*}$ An asterisk $\left(^{*}\right)$ indicates significance at the 0.05 level by one-way ANOVA and Dunnett's method, compared with GUS transgenic plants using JMP.

\section{Discussion}

Fusarium species employ TRI101 encoding trichothecene 3-O-acetyltransferase for self-protection from trichothecene mycotoxins [9]. In this study, we generated transgenic Arabidopsis plants expressing FgTri101 and demonstrated that these transgenic plants had enhanced resistance to several trichothecenes compared to Arabidopsis Col-0 and GUS transgenic plants. We showed that FgTri101 transgenic plants converted DON, 15-ADON, and NX-2 to their acetylated forms. Interestingly, we observed that 3-ADON, 3,15-diADON, and NX-3 were efficiently excreted into the media from FgTri101 transgenic plants. This study demonstrated that the overexpression of FgTri101 provides plant protection from important mycotoxins by detoxification and excretion. Further characterization of the transporters involved in toxin efflux will provide novel targets for generating transgenic crops to enhance FHB resistance and reduce mycotoxin contamination.

Gene expression analysis revealed varying levels of FgTri101 expression in the different transgenic Arabidopsis plants. Among four selected transgenic lines, the gene expression of FgTri101 was 700-fold higher in the line Tri101-12 than Tri101-10. Although the phenotypes of the four FgTri101 lines in root growth assays using $10 \mathrm{mg} / \mathrm{L}$ individual toxins were not significantly different, Tri101-10, which had the lowest FgTri101 expression, showed relatively low toxin conversion rates compared to Tri101-8, 12, and 17. A good correlation was demonstrated between the levels of lactoferrin protein produced in transgenic wheat lines and the level of resistance against $F$. graminearum [17]. Further investigations are needed to generate FgTri101 antibodies and use Western blots to quantify the levels of the FgTri101 protein.

The transgenic Arabidopsis plants expressing FgTri101 displayed enhanced resistance to trichothecenes, such as DON and NX-3, with longer roots and higher growth weights. Trichothecenes are phytotoxic and affect plant morphology, such as causing inhibition of root elongation and dwarfism [18]. Plant root growth inhibition assays are commonly used to evaluate the direct phytotoxic effects of trichothecenes. DON and other structural analogs have been shown to inhibit plant growth $[19,20]$. Some studies showed that 3ADON is highly toxic to wheat [21]. However, transgenic rice expressing FgTri101 displayed enhanced DON tolerance [13]. We showed that expression of FgTri101 not only increased Arabidopsis tolerance to DON, but also enhanced plant resistance toward NX-3 that is produced by a newly identified F. graminearum population (NA3). More importantly, we 
discovered that the acetylated trichothecenes were pumped out of plant cells, which may greatly reduce their phytotoxic effects. It will be interesting to determine whether the high toxicity caused by 3-ADON in wheat is due to a lack of transporters for 3-ADON excretion.

Our study demonstrated that approximately $89-98 \%$ of converted 3-ADON, 3,15diADON, or NX-2 by Tri101 transgenic Arabidopsis was excreted into the media. Since 3-ADON and 3,15-di ADON have been shown to be phytotoxic to plants [20], the enhanced root growth of FgTri101 transgenic plants may be due to most of the acylated trichothecenes being exported out of plant cells. Plant ATP-binding cassette (ABC) transporters are involved in toxin transportation and detoxification. For self-protection, many toxic metabolites are conjugated and transported to the plant vacuole for detoxification and sequestration [22]. Studies demonstrated that wheat plants contain two vacuolar membrane-localized transporters, TaABCC 3.1 and TaABCC3.2, which are induced by DON in wheat and associated with DON tolerance [23]. Although lacking direct evidence of TaABCC3.1 in DON transport and detoxification, the TaABCC3.1 transporter has been demonstrated to contribute to FHB resistance in wheat [23]. Arabidopsis AtABCC3, the closest homologue of TaABCC3 proteins, has been shown to localize within the vacuolar membrane (tonoplast) and transport glutathione conjugates and chlorophyll catabolites $[24,25]$. It is possible that AtABCC3 functions in DON sequestration. Most of the ABC transporters have been characterized to localize in the vacuolar membranes and sequester toxins. For example, PDR5 from Saccharomyces cerevisiae is a multidrug transporter [26] and TRI12 from F. sporotrichioides is a trichothecenes efflux pump for fungus self-protection [27]. In the Arabidopsis genome, from a total of 130 annotated ABC transporters, about 20 of them have been functionally characterized. In addition to sequestering toxins in vacuoles, there are many $\mathrm{ABC}$ transporters involved in detoxification and transportation of various compounds [28]. A plant plasma membrane ABC transporter, NpABC1, has been identified and is involved in the secretion of an antifungal terpenoid that plays a role in plant defense [29]. To the best of our knowledge, this is the first report showing trichothecenes excreted out of plants. Further investigations are needed to determine whether wheat and barley plants expressing FgTri101 have the ability to excrete acetylated toxins. We plan to identify the transporter/transporters that are involved in trichothecene excretion from plant cells, which may provide a novel target to reduce FHB and mycotoxin contamination.

FgTri101 and GUS transgenic Arabidopsis displayed similar healthy root length and growth weight when NIV was added, whereas GUS transgenic plants were significantly inhibited when DON or NX-3 was added. These observations suggest NIV is less phytotoxic to plants. Similarly, our recent study showed that DON, 3-ADON, and 15-ADON, but not NIV, inhibit wheat root elongation [20]. We did not observe NIV acetylation in transgenic Arabidopsis plants expressing FgTri101. F. graminearum strains produce NIV and acetylated NIV (typically 4,15-diANIV and 4-ANIV) in cultures [30]. To determine if Tri101 proteins are conserved between DON-producing strains and NIV-producing strains, we compared FgTri101 from PH-1 (used in this study for generation of Arabidopsis transgenic plants) and Tri101 from selected NIV strains. These Tri101 proteins share high identity and the catalytic histidine (FgH156 and 160D). We did observe that two amino acids were substituted in selected NIV strains, including A215P and L392F (Figure S6). The motif DFGWG(FgD389G393) is conserved in Fusarium species. However, the fourth position (Fg392) was observed as a Phe (F) in FsTRi101, while it is a Leu (L) in FgTri101 [14]. In comparison, we observed that 392F was present in all NIV-procuring Fusarium species (Figure S6). This suggests that the DFGWG motif may be important for substrate specificity. Further study is needed to determine if 392F is important for FgTri101 to bind NIV.

Regarding the effects of 3-ADON on the growth of cereal plants, there are conflicting results. Several studies show that 3-ADON is highly toxic to wheat at low concentrations [21]. On the other hand, studies find that 3-ADON does not inhibit rice root growth [13]. Ohsato and associates suggested that the higher toxicity of 3-ADON observed in cereal plants may be due to the instability of the C3 acetyl form in plant cells. In our study, we observed most of the 3-ADON was excreted out from plant cells, which greatly reduced 3-ADON 
phytotoxicity. More interestingly, while we only detected a trace amount of 3,15-diADON in a few transgenic Arabidopsis samples, we observed significant amounts of 3,15-diADON in the media. This suggests that either 3,15-diADON is unstable inside of plant cells, or it is quickly transported out of plant cells. We speculate that transgenic plants expressing FgTri101 may constantly export C-3 acetylated trichothecenes out of plant cells and protect crops from the phytotoxic effect of trichothecenes. Transgenic wheat and barley plants expressing FsTRI101 converted DON to an acetylated form, 3-ADON, but did not display effective FHB resistance [11,12]. This may be because FsTRI101 is less efficient in the conversion of DON to 3-ADON than FgTri101 [14]. It is also possible that wheat and barley lack transporters that excrete acetylated trichothecenes. Further investigations are needed to determine whether gene stacking of FgTri101 and the transporters for acylated toxin excretion provide effective protection for wheat and barley toward FHB and mycotoxin contamination.

Acetylation at the C-3 position has been shown to significantly reduce the phytotoxicity of a number of trichothecenes [31,32]. Therefore, expressing fungal Tri101 transacetylase could improve plant resistance to the toxins. This approach may work with other mycotoxins, but it would require different detoxification genes.

In conclusion, we demonstrated that transgenic Arabidopsis plants expressing FgTri101 displayed resistance to trichothecenes and converted several important trichothecenes to their less toxic acylated forms. More importantly, we showed that the acylated trichothecenes were excreted into the media from transgenic Arabidopsis plants expressing FgTri101. Further identification of the transporters for trichothecene excretion will provide novel breeding targets to reduce FHB and mycotoxin contamination.

\section{Materials and Methods}

\subsection{Construction of FgTri101 Vector and Arabidopsis Transformation}

Arabidopsis thaliana (ecotype Columbia, Col-0) were grown in a growth chamber at $23 / 20{ }^{\circ} \mathrm{C}$ with a $16 / 8 \mathrm{~h}$ light/dark cycle. The $\mathrm{pBinARS/plus} \mathrm{vector} \mathrm{was} \mathrm{used} \mathrm{for}$ FgTri101 vector construction [33]. FgTri101 contains no intron and was amplified using $F$. graminearum genomic DNA with primers FgTri101-ORF5' and ORF3', including the two enzyme cut sites Spe I and Xba I (Table S2). The PCR products of FgTri101 were purified, digested, and ligated into $\mathrm{pBinARS/plus} \mathrm{treated} \mathrm{with} \mathrm{the} \mathrm{same} \mathrm{enzymes.} \mathrm{The} \mathrm{ligation}$ mixture was used to transform TOP10 competent cells (Invitrogen, Carlsbad, CA, USA). The positive clone containing FgTri101 was obtained and sequenced. The purified plasmids of pBinARS/plus-FgTri101 driven by a double 35S (D35S) promoter were introduced into Agrobacterium tumefaciens strain GV2260 for Arabidopsis transformation.

Arabidopsis transformation was performed with pBinARS/plus-FgTri101 using floral dip methods [34]. Similarly, the pBinARS/plus vector carrying $\beta$-glucuronidase (GUS) was used to generate transgenic Arabidopsis plants as controls. Transgenic plants were selected on Murashige and Skoog (MS) media with the addition of $50 \mathrm{mg} / \mathrm{L}$ kanamycin. After about two weeks, the kanamycin-resistant plants were transferred to soil and grown in the growth chamber as described above.

\subsection{Molecular Analysis of Transgenic Arabidopsis}

Plant genomic DNA was isolated from leaves of kanamycin-resistant plants using ZR Fungal/Bacterial DNA miniprep kit (Zymo Research, Boston, MA, USA). DNA concentration and quality were evaluated using a spectrophotometer (NanoDrop 2000, Thermofisher Scientific, Waltham, MA, USA). PCR was used to amplify the target gene using the primers D35S-F and Nos-R (Table S2).

Total RNA was extracted from leaves of transgenic Arabidopsis plant containing FgTri101 using a Trizol reagent (Sigma-Aldrich, St. Louis, MO, USA) combined with the Ambion RNA isolation kit [35]. RNA was quantified with a spectrophotometer (Nanodrop; Thermo Fisher Scientific, Waltham, MA, USA) and treated with RQ1 RNase-free DNase (Promega Corp. Madison, WI, USA). The absence of genomic DNA contamination 
was verified by quantitative PCR (qPCR). The first-strand cDNA was synthesized, and reverse transcriptase qPCR (RT-qPCR) was performed on a Bio-Rad CFX96 real-time system (Bio-Rad Laboratories, Hercules, CA, USA) using primers FgTri101-RT-5' and FgTri101-RT-3' (Table S2). The Arabidopsis gene elongation factor 1-alpha (EF1 $\alpha$ ) was used to normalize the values as an internal control. The gene expression level was calculated with the $2^{-\Delta \Delta \mathrm{Ct}}$ method using CFX manager software (Bio-Rad), relative to the transgenic plant with the lowest expression level (Tri101-10), which was set as 1 . The qPCR reactions were set up in triplicate and repeated three times with similar results.

To obtain homozygotes for further analysis, T2 seeds from transgenic lines were sown on MS media containing $50 \mathrm{mg} / \mathrm{L}$ kanamycin for a progeny test. The transgenic lines for which T2 seedlings showed no segregation were considered as homozygotes. The FgTri101 copy number was estimated by qPCR in homozygous T2 lines Tri101-8, 12, 17 and all $\mathrm{T}_{0}$ transgenic lines as described $[35,36]$. The Arabidopsis gene 4-hydroxyphenypyruvate dioxygenase (4HPPD), which is a single-copy gene, was used as the endogenous reference gene [36]. Primer efficiency for FgTri101 and $4 H P P D$ was determined using a standard curve consisting of a dilution series for each primer pair. The qPCR amplification efficiency was calculated according to the following equation: Efficiency $=10^{(-1 / \text { slope })}-1$. The ratio of the copy number of TRI101 was calculated using the following equation: Ratio $=(1+$ Efficiency

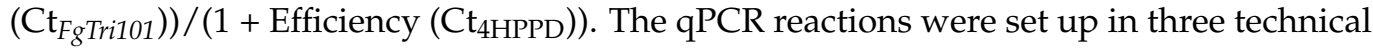
triplicates and repeated three times. Data are shown as means \pm SD of three replicates.

\subsection{Mycotoxins}

DON, 15-ADON, NIV, and NX-3 were purified at the Mycotoxin Prevention and Applied Microbiology Research Unit, USDA/ARS, Peoria, IL. DON, NX-3, and NIV were dissolved in water at $5 \mathrm{mg} / \mathrm{mL}$. 15-ADON was dissolved in $200 \mu \mathrm{l}$ methanol and water was added to a final concentration of $5 \mathrm{mg} / \mathrm{mL}$.

\subsection{Root Growth Assays}

Although F. graminearum produces the acetylated trichothecenes in culture, e.g., 3$\mathrm{ADON}, 15-\mathrm{ADON}$, and NX-2, most of these toxins are converted to their deacetylated forms, DON, NIV, and NX-3 in planta. Therefore, root growth assays were conducted on a MS medium with the addition of DON, NIV, and NX-3 in square vertical plates (Greiner bio-one North America Inc. Monroe, NC).

$\mathrm{T}_{2}$ seeds of the transgenic plants expressing GUS or FgTri101 (lines 8,10,12, and 17) were surface sterilized using 70\% ethanol for $2 \mathrm{~min}$, then $50 \%$ Clorox for $10 \mathrm{~min}$, and rinsed three times with sterile water. The seeds were sown on MS medium containing $10 \mathrm{mg} / \mathrm{L}$ DON, NIV, or NX-3. A total of nine surface-sterilized seeds were placed on each agar plate. Four plates from each transgenic line were treated with each toxin. The plates were placed vertically in a growth chamber as described above. The root length was measured every 2 days for 12 days after germination. Root length $(n=36-40)$ from each transgenic line was analyzed and compared with GUS controls using JMP.

\subsection{Conversion of Trichothecenes by Transgenic Arabidopsis Seedlings}

$\mathrm{T}_{2}$ seeds of the transgenic plants expressing GUS and FgTri101 (lines 8, 10, 12, and 17) were surface sterilized as described above. The sterilized seeds were placed on a MS medium containing $50 \mathrm{mg} / \mathrm{L}$ kanamycin. The plates were kept at $4{ }^{\circ} \mathrm{C}$ for 2 days and moved to a growth chamber at $23 / 20^{\circ} \mathrm{C}$ in a $16 / 8 \mathrm{~h}$ light/dark cycle. After $7-8$ days, about 16 plants were placed into $50 \mathrm{~mL}$ tubes to grow in $5 \mathrm{~mL}$ of half-strength MS liquid medium containing $1 \%$ sucrose. After 3-4 days, the media were removed and substituted with $5 \mathrm{~mL}$ of fresh medium plus $50 \mathrm{mg} / \mathrm{L}$ DON, 15-ADON, NIV, or NX-3, respectively. The samples were collected two days after toxin treatments. The seedlings were rinsed three times with $5 \mathrm{~mL}$ of water to remove external toxins. The rinsed water was combined with the media for toxin measurement. The fresh weigh of seedlings was measured. The seedling samples and culture media were used for toxin analysis. Each transgenic line was treated with 
three biological replicates for each toxin. Media containing toxins without plants served as controls.

\subsection{Mycotoxin Extraction and Quantification}

Arabidopsis seedlings were extracted with $10 \mathrm{~mL}$ of acetonitrile:water (86:14) by shaking for $1 \mathrm{~h}$ at $250 \mathrm{rpm}$ on an orbital shaker. The samples were centrifuged for $5 \mathrm{~min}$ at $4000 \mathrm{rpm}$, and $9 \mathrm{~mL}$ of extract was dried under a stream of nitrogen [37]. For liquid media, the toxin was extracted from $1.4 \mathrm{~mL}$ aliquots from $20 \mathrm{~mL}$ that were combined with $8.6 \mathrm{~mL}$ acetonitrile, and the mixture was dried under a stream of nitrogen.

Trimethylsilyl (TMS) derivatives were prepared by adding $100 \mu \mathrm{L}$ of a 100:1 freshly prepared mixture of N-trimethylsilylimadazole/trimethylchlorosilane (Sigma-Aldrich, St. Louis, MO, USA) to the dried extract. After $30 \mathrm{~min}, 900 \mu \mathrm{L}$ of isooctane was added to the reaction mixture followed by $1 \mathrm{~mL}$ water. The organic layer was transferred to $2-\mathrm{mL}$ autosampler vial for GC-MS analysis. TMS derivatives of purified DON, 3-ADON, 15ADON, 3,15-diADON, NX-2, and NX-3 (0.3125 $\mu \mathrm{g}$ to $80 \mu \mathrm{g})$ were similarly prepared and used to construct standard curves for quantification.

GC-MS analyses were performed on an Agilent 7890 gas chromatograph fitted with a HP-5MS column ( $30 \mathrm{~m}, 0.25 \mathrm{~mm}, 0.25 \mu \mathrm{m})$ with splitless injection and a 5977 mass detector. The injection temperature was kept at $250^{\circ} \mathrm{C}$ and the column flow rate was $1 \mathrm{~mL} \mathrm{~min}{ }^{-1}$. A temperature program was used with initial column temperature of $150^{\circ} \mathrm{C}$ for $1 \mathrm{~min}$, and then increased to $280^{\circ} \mathrm{C}$ at $30^{\circ} \mathrm{C} \mathrm{min}^{-1}$ and held for $3.5 \mathrm{~min}$. Samples were analyzed in both scan and selective ion monitoring (SIM) mode. Under these conditions, the TMS ether of DON was detected at $6.14 \mathrm{~min}(512,422,392,295,259$, and 235 ions), 3ADON at $6.60 \mathrm{~min}(482,467,392,377,235,193$, and 181 ions), $15 \mathrm{ADON}$ at $6.70 \mathrm{~min}(482,467,392,235$, 193, and 181 ions), 3,15-diADON at $7.23 \mathrm{~min}$ (437, 377, 320, 262, 233, and 193 ions), NX-3 at $6.04 \mathrm{~min}(408,305$, and 181 ions), NX-2 at $6.56 \mathrm{~min}(378,305,275$, and 181 ions), and NIV at 6.81 (510, 482, 289, 191, and 103 ions). Mass Hunter software with a NIST11 library was used to identify additional peaks in the chromatograms.

The total excreted toxin was calculated by the following formula: 3-acetylated toxin $x$ $20 / 1.4$, and divided by the total acetylated toxin in the plant and media.

\subsection{Statistical Analyses}

All statistical analyses were performed using JMP software. Individual analyses are discussed in each Section 4.

Supplementary Materials: The following are available online at https:/ / www.mdpi.com/article/ 10.3390/toxins13050320/s1, Figure S1: (a) Diagram of FgTri101 construct in the binary vector pBinARS/PLUS. (b) PCR amplification of FgTri101 gene from transgenic Arabidopsis plants. Figure S2 Arabidopsis seedling growth on MS media containing different trichothecenes $(10 \mathrm{mg} / \mathrm{L})$. Figure S3 Root length comparison after a two-week incubation on MS media containing toxins. Figure S4: Chromatographs show 15-ADON converted to 3,15-ADON by FgTri101 transgenic seedlings, but not in GUS controls. Figure S5: Chromatographs show no detectable acetylated NIV by FgTri101 transgenic Arabidopsis. Figure S6: Comparison of deduced Tri101 amino acid sequences from DON producing strain PH-1 and NIV producing strains from Fusarium family. Table S1: The copy number of FgTri101 in transgenic Arabidopsis. Table S2: Primers used in the study.

Author Contributions: Conceptualization, G.H. and S.M.; Methodology, G.H., H.T., T.U. and S.M.; Data analysis, G.H. and H.T.; Writing—original draft preparation, G.H.; Writing—review and editing, G.H., H.T. and S.M. All authors have read and agreed to the published version of the manuscript.

Funding: This research was supported by the U.S. Department of Agriculture, Agricultural Research Service.

Institutional Review Board Statement: Not applicable.

Informed Consent Statement: Not applicable.

Data Availability Statement: Not applicable. 
Acknowledgments: We thank Stephanie Folmar for her technical support. This work was supported in part by the U.S. Department of Agriculture, Agricultural Research Service. Mention of trade names or commercial products in this publication is solely for the purpose of providing specific information and does not imply recommendation or endorsement by the U.S. Department of Agriculture. USDA is an equal opportunity provider and employer.

Conflicts of Interest: The authors declare no conflict of interest.

\section{References}

1. Rocha, O.; Ansari, K.; Doohan, F.M. Effects of trichothecene mycotoxins on eukaryotic cells: A review. Food Addit. Contam. 2005, 22, 369-378. [CrossRef] [PubMed]

2. Bin-Umer, M.A.; McLaughlin, J.E.; Basu, D.; McCormick, S.; Tumer, N.E. Trichothecene Mycotoxins Inhibit Mitochondrial Translation-Implication for the Mechanism of Toxicity. Toxins 2011, 3, 1484-1501. [CrossRef] [PubMed]

3. Proctor, R.H. Reduced Virulence ofGibberella zeaeCaused by Disruption of a TrichotheceneToxin Biosynthetic Gene. Mol. Plant Microbe Interact. 1995, 8, 593-601. [CrossRef] [PubMed]

4. Jansen, C.; Von Wettstein, D.; Schäfer, W.; Kogel, K.-H.; Felk, A.; Maier, F.J. Infection patterns in barley and wheat spikes inoculated with wild-type and trichodiene synthase gene disrupted Fusarium graminearum. Proc. Natl. Acad. Sci. USA 2005, 102, 16892-16897. [CrossRef] [PubMed]

5. Shin, S.; Torres-Acosta, J.A.; Heinen, S.J.; McCormick, S.; Lemmens, M.; Paris, M.P.K.; Berthiller, F.; Adam, G.; Muehlbauer, G.J. Transgenic Arabidopsis thaliana expressing a barley UDP-glucosyltransferase exhibit resistance to the mycotoxin deoxynivalenol. J. Exp. Bot. 2012, 63, 4731-4740. [CrossRef] [PubMed]

6. $\quad$ Li, X.; Shin, S.; Heinen, S.; Dill-Macky, R.; Berthiller, F.; Nersesian, N.; Clemente, T.; McCormick, S.; Muehlbauer, G.J. Transgenic Wheat Expressing a Barley UDP-Glucosyltransferase Detoxifies Deoxynivalenol and Provides High Levels of Resistance to Fusarium graminearum. Mol. Plant Microbe Interact. 2015, 28, 1237-1246. [CrossRef] [PubMed]

7. Wang, H.; Sun, S.; Ge, W.; Zhao, L.; Hou, B.; Wang, K.; Lyu, Z.; Chen, L.; Xu, S.; Guo, J.; et al. Horizontal gene transfer of Fhb7 from fungus underlies Fusarium head blight resistance in wheat. Science 2020, 368, eaba5435. [CrossRef]

8. Proctor, R.H.; McCormick, S.P.; Kim, H.-S.; Cardoza, R.E.; Stanley, A.M.; Lindo, L.; Kelly, A.; Brown, D.W.; Lee, T.; Vaughan, M.M.; et al. Evolution of structural diversity of trichothecenes, a family of toxins produced by plant pathogenic and entomopathogenic fungi. PLoS Pathog. 2018, 14, e1006946. [CrossRef] [PubMed]

9. McCormick, S.P.; Alexander, N.J.; Trapp, S.E.; Hohn, T.M. Disruption of TRI101, the Gene Encoding Trichothecene 3-OAcetyltransferase, fromFusarium sporotrichioides. Appl. Environ. Microbiol. 1999, 65, 5252-5256. [CrossRef]

10. Muhitch, M.J.; McCormick, S.P.; Alexander, N.J.; Hohn, T.M. Transgenic expression of the TRI101 or PDR5 gene increases resistance of tobacco to the phytotoxic effects of the trichothecene 4,15-diacetoxyscirpenol. Plant Sci. 2000, 157, 201-207. [CrossRef]

11. Okubara, P.A.; Blechl, A.E.; McCormick, S.P.; Alexander, N.J.; Dill-Macky, R.; Hohn, T.M. Engineering deoxynivalenol metabolism in wheat through the expression of a fungal trichothecene acetyltransferase gene. Theor. Appl. Genet. 2002, 106, 74-83. [CrossRef]

12. Manoharan, M.; Dahleen, L.S.; Hohn, T.M.; Neate, S.M.; Yu, X.-H.; Alexander, N.J.; McCormick, S.P.; Bregitzer, P.; Schwarz, P.B.; Horsley, R.D. Expression of 3-OH trichothecene acetyltransferase in barley (Hordeum vulgare L.) and effects on deoxynivalenol. Plant Sci. 2006, 171, 699-706. [CrossRef]

13. Ohsato, S.; Ochiai-Fukuda, T.; Nishiuchi, T.; Takahashi-Ando, N.; Koizumi, S.; Hamamoto, H.; Kudo, T.; Yamaguchi, I.; Kimura, M. Transgenic rice plants expressing trichothecene 3-O-acetyltransferase show resistance to the Fusarium phytotoxin deoxynivalenol. Plant Cell Rep. 2006, 26, 531-538. [CrossRef] [PubMed]

14. Garvey, G.S.; McCormick, S.P.; Rayment, I. Structural and Functional Characterization of the TRI101 Trichothecene 3-OAcetyltransferase from Fusarium sporotrichioides and Fusarium graminearum. J. Biol. Chem. 2008, 283, 1660-1669. [CrossRef] [PubMed]

15. Kelly, A.C.; Ward, T.J. Population genomics of Fusarium graminearum reveals signatures of divergent evolution within a major cereal pathogen. PLoS ONE 2018, 13, e0194616. [CrossRef]

16. Woelflingseder, L.; Gruber, N.; Adam, G.; Marko, D. Pro-Inflammatory Effects of NX-3 Toxin Are Comparable to Deoxynivalenol and not Modulated by the Co-Occurring Pro-Oxidant Aurofusarin. Microorganisms 2020, 8, 603. [CrossRef]

17. Han, J.; Lakshman, D.K.; Galvez, L.C.; Mitra, S.; Baenziger, P.S.; Mitra, A. Transgenic expression of lactoferrin imparts enhanced resistance to head blight of wheat caused by Fusarium graminearum. BMC Plant Biol. 2012, 12, 33. [CrossRef]

18. Masuda, D.; Ishida, M.; Yamaguchi, K.; Yamaguchi, I.; Kimura, M.; Nishiuchi, T. Phytotoxic effects of trichothecenes on the growth and morphology of Arabidopsis thaliana. J. Exp. Bot. 2007, 58, 1617-1626. [CrossRef]

19. Gatti, M.; Choulet, F.; Macadré, C.; Guérard, F.; Seng, J.-M.; Langin, T.; Dufresne, M. Identification, Molecular Cloning, and Functional Characterization of a Wheat UDP-Glucosyltransferase Involved in Resistance to Fusarium Head Blight and to Mycotoxin Accumulation. Front. Plant Sci. 2018, 9, 1853. [CrossRef]

20. Wipfler, R.; McCormick, S.P.; Proctor, R.H.; Teresi, J.M.; Hao, G.; Ward, T.J.; Alexander, N.J.; Vaughan, M.M. Ward Synergistic Phytotoxic Effects of Culmorin and Trichothecene Mycotoxins. Toxins 2019, 11, 555. [CrossRef]

21. Eudes, F.; Comeau, A.; Rioux, S.; Collin, J. Phytotoxicité de huit mycotoxines associées à la fusariose de l'épi chez le blé. Can. J. Plant Pathol. 2000, 22, 286-292. [CrossRef] 
22. Cummins, I.; Dixon, D.P.; Freitag-Pohl, S.; Skipsey, M.; Edwards, R. Multiple roles for plant glutathione transferases in xenobiotic detoxification. Drug Metab. Rev. 2011, 43, 266-280. [CrossRef]

23. Walter, S.; Kahla, A.; Arunachalam, C.; Perochon, A.; Khan, M.R.; Scofield, S.R.; Doohan, F.M. A wheat ABC transporter contributes to both grain formation and mycotoxin tolerance. J. Exp. Bot. 2015, 66, 2583-2593. [CrossRef]

24. Tommasini, R.; Vogt, E.; Fromenteau, M.; Hörtensteiner, S.; Matile, P.; Amrhein, N.; Martinoia, E. An ABC-transporter of Arabidopsis thalianahas both glutathione-conjugate and chlorophyll catabolite transport activity. Plant J. 1998, 13, 773-780. [CrossRef]

25. Szponarski, W.; Sommerer, N.; Boyer, J.-C.; Rossignol, M.; Gibrat, R. Large-scale characterization of integral proteins from Arabidopsis vacuolar membrane by two-dimensional liquid chromatography. Proteomics 2004, 4, 397-406. [CrossRef] [PubMed]

26. Balzi, E.; Wang, M.; Leterme, S.; Van Dyck, L.; Goffeau, A. PDR5, a novel yeast multidrug resistance conferring transporter controlled by the transcription regulator PDR1. J. Biol. Chem. 1994, 269, 2206-2214. [CrossRef]

27. Alexander, N.J.; McCormick, S.P.; Hohn, T.M. TRI12, a trichothecene efflux pump from Fusarium sporotrichioides: Gene isolation and expression in yeast. Mol. Genet. Genom. 1999, 261, 977-984. [CrossRef] [PubMed]

28. Kang, J.; Park, J.; Choi, H.; Burla, B.; Kretzschmar, T.; Lee, Y.; Martinoia, E. Plant ABC Transporters. Arab. Book 2011, 9, e0153. [CrossRef] [PubMed]

29. Jasinski, M.; Stukkens, Y.; Degand, H.; Purnelle, B.; Marchand-Brynaert, J.; Boutry, M. A plant plasma membrane ATP binding cassette-type transporter is involved in antifungal terpenoid secretion. Plant Cell 2001, 13, 1095-1107. [CrossRef]

30. Lee, T.; Lee, S.-H.; Shin, J.Y.; Kim, H.-K.; Yun, S.-H.; Kim, H.-Y.; Lee, S.; Ryu, J.-G. Comparison of Trichothecene Biosynthetic Gene Expression between Fusarium graminearum and Fusarium asiaticum. Plant Pathol. J. 2014, 30, 33-42. [CrossRef]

31. Alexander, N.J.; McCormick, S.P.; Ziegenhorn, S.L. Phytotoxicity of selected trichothecenes using Chlamydomonas rein-hardtii as a model systemt. Nat. Toxins 1999, 7, 265-269. [CrossRef]

32. Desjardins, A.E.; McCormick, S.P.; Appell, M. Structure-Activity Relationships of Trichothecene Toxins in anArabidopsis thalianaLeaf Assay. J. Agric. Food Chem. 2007, 55, 6487-6492. [CrossRef] [PubMed]

33. Hao, G.; Stover, E.; Gupta, G. Overexpression of a Modified Plant Thionin Enhances Disease Resistance to Citrus Canker and Huanglongbing (HLB). Front. Plant Sci. 2016, 7, 1078. [CrossRef]

34. Clough, S.J.; Bent, A.F. Floral dip: A simplified method for Agrobacterium-mediated transformation of Arabidopsis thaliana. Plant J. 1998, 16, 735-743. [CrossRef]

35. Hao, G.; Bakker, M.G.; Kim, H.S. Enhanced Resistance to Fusarium graminearum in Transgenic Arabidopsis Plants Expressing a Modified Plant Thionin. Phytopathology 2020. [CrossRef] [PubMed]

36. Garcia, I.; Rodgers, M.; Pepin, R.; Hsieh, T.-F.; Matringe, M. Characterization and Subcellular Compartmentation of Recombinant 4-Hydroxyphenylpyruvate Dioxygenase from Arabidopsis in Transgenic Tobacco. Plant Physiol. 1999, 119, 1507-1516. [CrossRef]

37. Hao, G.; McCormick, S.; Vaughan, M.M.; Naumann, T.A.; Kim, H.-S.; Proctor, R.; Kelly, A.; Ward, T.J. Fusarium graminearum arabinanase (Arb93B) Enhances Wheat Head Blight Susceptibility by Suppressing Plant Immunity. Mol. Plant Microbe Interact. 2019, 32, 888-898. [CrossRef] [PubMed] 